\title{
A finite number of point observations which determine a non-autonomous fluid flow
}

\author{
José A Langa ${ }^{1}$ and James C Robinson ${ }^{2}$ \\ ${ }^{1}$ Departamento de Ecuaciones Diferenciales y Análisis Numérico, Universidad de Sevilla, \\ Apdo de Correos 1160, 41080-Sevilla, Spain \\ 2 Mathematics Institute, University of Warwick, Coventry CV4 7AL, UK \\ E-mail: langa@numer.us.es and jcr@maths.warwick.ac.uk
}

Received 19 June 2000

Recommended by F Otto

\begin{abstract}
We show that a finite number of point observations serve to determine the flow field throughout the entire domain for certain two-dimensional (2D) flows. In particular, we consider the 2D Navier-Stokes equations with periodic boundary conditions and a time-dependent forcing which is analytic in space. Using the theory of non-autonomous attractors developed by Chepyzhov and Vishik, and the theory of point observations developed by Friz and Robinson, we show that almost every choice of a sufficient number of 'nodes' in the domain gives an evaluation map $u \mapsto\left(u\left(x_{1}\right), \ldots, u\left(x_{k}\right)\right)$ which is one-to-one between the attractor and its image.
\end{abstract}

Mathematics Subject Classification: 35B40, 35B42, 35Q30, 37L30, 76D05

\section{Introduction}

The reduction of the dynamics of infinite-dimensional dissipative equations to a finitedimensional system is a topic which has received much attention, and been the subject of a variety of approaches. The surprising fact that many of these a priori infinite-dimensional systems have a finite-dimensional global attractor is now well attested. Indeed, proof of the existence of such attractors for an ever-expanding array of models is continually swelling the literature. (Temam (1988) contains many examples, and the second edition of the same book (1996) yet more.) However, that the attractor is finite-dimensional is not a statement about the dynamics of the equation, but only about the properties of the attractor as a subset of the phase space. To translate this into some information about the dynamics requires different techniques.

In this paper we focus on the two-dimensional (2D) Navier-Stokes equations, to which the above theory applies. Indeed, many of the most important concepts have been introduced in the context of this equation. For example, the most powerful method for estimating the dimension of attractors was introduced by Constantin and Foias (1985) in a paper which provided bounds 
on this dimension for the Navier-Stokes equation with both Dirichlet and periodic boundary conditions. Although here we restrict our attention to the periodic case, similar arguments work for the Dirichlet case.

Some of the techniques for dealing with finite-dimensional dynamics appear to be independent of the existence of the attractor. As early as 1967 Foias and Prodi proved that, for the 2D Navier-Stokes equations, a finite number of Fourier modes are 'asymptotically determining', in that if

$$
\left|P_{n} u(t)-P_{n} v(t)\right| \rightarrow 0
$$

then

$$
|u(t)-v(t)| \rightarrow 0
$$

(Here, $P_{n}$ represents the projection of the solution onto the first $n$ eigenvalues of the Stokes operator.) A related idea is that of 'determining nodes' due to Foias and Temam (1984), in which condition (1.1) is replaced by

$$
\max _{j=1, \ldots, N}\left|u\left(x_{j}, t\right)-v\left(x_{j}, t\right)\right| \rightarrow 0
$$

where $\left\{x_{j}\right\}$ is a set of points spaced sufficiently closely throughout the spatial domain. These and other similar ideas have been developed further by various authors (see Cockburn et al (1997) for a general framework into which both of these fit, and for more references).

A more recent approach, the theory of inertial manifolds (see Foias et al (1988), and many papers thereafter-Temam (1988) and Robinson (2001b) both contain a selection of references), seeks to find a finite-dimensional Lipschitz manifold containing the global attractor, which one can use to (more or less) explicitly reduce the asymptotic dynamics to a finite-dimensional set of ordinary differential equations (the 'inertial form'). However, the conditions necessary to apply this theory are very restrictive, and in particular exclude the 2D Navier-Stokes equations.

Currently there are two direct approaches which make use of the global attractor itself. The first attempts to construct a finite-dimensional system which reproduces the dynamics on the attractor, aping the 'inertial form', but without recourse to an inertial manifold. This idea, pursued in Eden et al (1994) and Robinson (1999), is far from straightforward, and the solutions offered in both of these references have different problems (the first, a discontinuous nonlinearity and non-uniqueness of solutions; the second, restriction to the case of discrete time).

Strictly, the second direct approach does not immediately give information about the dynamics per se, but does indicate strongly that there is an underlying finite-dimensional system. Here the idea is to show that there is a finite set of quantities which parametrize the attractor. Since the first paper by Mañé (1981) this theory has also been developed by several authors, generally providing an 'abstract' parametrization (Eden et al 1994, Foias and Olson 1996, Hunt and Kaloshin 1999). However, recently Friz and Robinson (2001) (see also Friz et al 2001) proved a result providing a parametrization based on the physical domain rather than the phase space, showing that observations at a finite number of points in the domain can parametrize the attractor.

However, all of these parametrizations are usually restricted, along with the classical theory of global attractors, to the (perhaps unphysical) case of a time-independent forcing. In this paper we generalize the parametrization approach to the non-autonomous case.

In section 2 we briefly recall the definition of a global attractor for an autonomous system; we introduce the fractal (box-counting) dimension; and recall various results concerning 
parametrizations of finite-dimensional sets. In section 3 we discuss the theory of nonautonomous attractors, and show that there is an appropriate notion of their dimension, following Chepyzhov and Vishik (1993).

Using these ideas we generalize both the abstract parametrization result due to Hunt and Kaloshin (section 4), and the nodal parametrization of Friz and Robinson (section 5), to the non-autonomous case. For further discussion of the physical interpretation of such results, see Robinson (2001a).

\section{Classical theory for autonomous systems}

In order to be concrete we restrict our discussion to the 2D Navier-Stokes equations with periodic boundary conditions,

$$
\frac{\partial u}{\partial t}+(u \cdot \nabla) u+v \Delta u+\nabla p=g(t) \quad \nabla \cdot u=0
$$

for $x \in Q=[0, L]^{2}$. Standard manipulations (see Constantin and Foias 1988, Temam 1988, Robinson 2001b) transform this into an abstract evolution equation

$$
\mathrm{d} u / \mathrm{d} t+B(u, u)+v A_{s} u=f(t)
$$

on the Hilbert space

$$
H=\left\{u \in \mathbb{L}^{2}(Q): \nabla \cdot u=0\right\}
$$

Here, $A_{s}$ is the Stokes operator (the orthogonal projection of $-\Delta$ onto $H$ ) and $B(u, u)$ is a bilinear term derived from the nonlinearity $(u \cdot \nabla) u . \mathbb{L}^{2}(Q)=\left[L^{2}(Q)\right]^{2}$ is the space of two component vectors $\left(u_{1}, u_{2}\right)$ with $u_{i} \in L^{2}(Q)$.

Solutions of (2.1) can be used to define a semidynamical system on $H$, so that

$$
S(t) u_{0}=u\left(t ; u_{0}\right)
$$

is a continuous semigroup of transformations on $H$. By proving the existence of a compact absorbing set $B$ in $H$, i.e. a set such that

$$
S(t) D \subset B \quad \text { for all } t \geqslant t_{0}(X)
$$

for any bounded set $D \subset H$, one can show that the equation has a compact global attractor $\mathcal{A}$. $\mathcal{A}$ is invariant,

$$
S(t) \mathcal{A}=\mathcal{A} \quad \text { for all } \quad t \in \mathbb{R}
$$

and attracts all bounded sets,

$$
\operatorname{dist}(S(t) D, \mathcal{A}) \rightarrow 0 \quad \text { as } \quad t \rightarrow \infty
$$

(for general results on global attractors see Hale (1988), Ladyzhenskaya (1991), or Temam (1988)).

The fractal dimension of $\mathcal{A}, d_{f}(\mathcal{A})$, is defined as

$$
d_{f}(\mathcal{A})=\limsup _{\epsilon \rightarrow 0} \frac{\log N(\mathcal{A}, \epsilon)}{-\log \epsilon}
$$

where $N(\mathcal{A}, \epsilon)$ is the minimum number of balls of radius $\epsilon$ which is necessary to cover $\mathcal{A}$. The method developed by Constantin and Foias (1985) has been used to show that many attractors 
have finite fractal dimension. Our case, the 2D Navier-Stokes equations, was the subject of their paper (see also Doering and Gibbon 1995, Temam 1988).

The importance of a finite fractal dimension is highlighted by the following theorem due to Hunt and Kaloshin (1999) (see the introduction for references containing various related results). The content is that if $X$ has finite fractal dimension, 'most' linear maps into $\mathbb{R}^{k}$, with $k$ large enough, are one-to-one between $X$ and its image.

We recall here that if $X$ is compact, and $f: X \rightarrow f(X)$ is continuous and one-to-one, then $f^{-1}: f(X) \rightarrow X$ is also continuous. In this way, a result such as theorem 2.1 below also provides a parametrization of $X$ using $k$ coordinates.

We choose not to give a precise definition here of the idea of 'prevalence' used in the theorem, referring instead to Hunt et al (1992) for more details. Instead, we merely give two important properties from that paper. First, any prevalent set is dense (their fact $2^{\prime}$ ), and secondly, the countable intersection of prevalent sets is also prevalent (their fact $3^{\prime \prime}$ ). This shows clearly that the property of prevalence is much stronger than that of density. Indeed, there are good reasons for considering prevalence to be an appropriate notion of 'almost every' for infinite-dimensional spaces (see Hunt et al (1992) for a full discussion).

Theorem 2.1 (Hunt and Kaloshin). Let $X$ be a compact subset of $H$ with $d_{f}(X)<d$. Provided that $k>2 d$, a prevalent set of linear maps $L: H \rightarrow \mathbb{R}^{k}$ are one-to-one between $X$ and its image, and the map $L^{-1}: L X \mapsto X$ is Hölder continuous.

(The result is also valid for Banach spaces, and in its original form provides an explicit bound for the Hölder exponent based on the 'thickness' of $X$. Although as stated theorem 2.1 is very similar to that of Foias and Olson (1996), we will need the prevalence property in what follows.)

This theorem shows that, in some sense, 'most' choices of a linear map provide a $k$ dimensional parametrization of a finite-dimensional attractor. However, these are 'abstract' parametrizations which are not in any way a priori connected with the physical domain. In an attempt to remedy this, Friz and Robinson (2001) proved a conjecture due to Foias and Temam (1984) by making use of the spatial analyticity of functions on the attractor.

When the forcing term in the Navier-Stokes equations is analytic, so are the functions on the attractor. More precisely, if $A$ is the negative Laplacian, then if

$$
f \in D\left(\mathrm{e}^{\sigma A^{1 / 2}}\right)
$$

for some $\sigma>0$, the attractor is uniformly bounded in $D\left(\mathrm{e}^{\tau A^{1 / 2}}\right)$, for some $\tau>0 .\left(D\left(\mathrm{e}^{\sigma A^{1 / 2}}\right)\right.$ denotes the domain in $\mathbb{L}^{2}(Q)$ of $\mathrm{e}^{\sigma A^{1 / 2}}$.) This space, a Gevrey class of analytic functions (see Friz and Robinson (2001) for more details), we denote by the more compact notation $G_{\tau}(Q)$.

We have the following theorem, originally proved in Friz and Robinson (2001) and improved (by lowering the minimum value of $k$ ) in Friz et al (2001).

Theorem 2.2. Let $Q \subset \mathbb{R}^{n}$, and X be a compact subset of $\mathbb{L}^{2}(Q)=\left[L^{2}(Q)\right]^{n}$, with $d_{f}(X)<d$ and $X$ uniformly bounded in $G_{\tau}(Q)$. Then, provided that $k \geqslant 16 d+1$, for almost every set of $k$ nodes

$$
\left(x_{1}, \ldots, x_{k}\right) \in Q^{k}
$$

the map

$$
E_{x}[u]=\left(u\left(x_{1}\right), \ldots, u\left(x_{k}\right)\right)
$$

is one-to-one between $X$ and its image. In other words, point observations at the nodes $\left(x_{1}, \ldots, x_{k}\right)$ determine uniquely the element $u \in X$. ('Almost every' is with respect to nkdimensional Lebesgue measure.) 
As before, we can use the compactness of $X$ to deduce that the values of $u$ at the nodal points provide a parametrization of the attractor.

\section{Non-autonomous attractors}

We now discuss the theory of non-autonomous attractors as developed by Chepyzhov and Vishik (1993, 1994), although they do not use this terminology (a very similar construction appears in Crauel et al (1995), see also Schmalfuß (1999)). We adopt it since it emphasizes the relationship with autonomous attractors and the theory of random attractors due to Schmalfuß (1992), Crauel and Flandoli (1994) and Crauel et al (1995). The term 'cocycle attractor' has been used for these sets by Kloeden and Schmalfuß (1997).

When $f$ depends on time, the solutions of the Navier-Stokes equation (2.1) depend not only on the initial condition but also on the initial time, so that instead of the semigroup $S(t)$, we obtain a process $S(t, s)$, where

$$
S(t, s) u_{0}=u\left(t ; s, u_{0}\right)
$$

the solution at time $t$ of (2.1) with $u(s)=u_{0}$. We note here the Lipschitz property of $S(t, s)$,

$$
\left|S(t, s) u_{0}-S(t, s) v_{0}\right| \leqslant L(t-s)\left|u_{0}-v_{0}\right|
$$

which will be useful later.

A non-autonomous attractor is a collection of compact sets $\{\mathcal{A}(s): s \in \mathbb{R}\}$, the 'kernel sections' of Chepyzhov and Vishik, such that

$$
S(t, s) \mathcal{A}(s)=\mathcal{A}(t)
$$

and, for any bounded set $D$,

$$
\lim _{s \rightarrow-\infty}(S(t, s) D, \mathcal{A}(t))=0 \quad \text { for all } t \in \mathbb{R} .
$$

This 'pullback convergence' property means that if we observe an experiment now (time $t$ ) which we know has already been running for a long time (since time $s$ ), we can expect that its state lies close to the set $\mathcal{A}(t)$. The invariance property (3.2) means that it still makes sense to discuss the dynamics 'restricted to the attractor'.

Conditions for the existence of such a set are similar to those in the deterministic case. In particular, if there exists a compact set $B$ which is 'uniformly attracting', so that, for any bounded set $D$,

$$
\operatorname{dist}(S(t+s ; s) D, B) \rightarrow 0 \quad \text { as } \quad t \rightarrow \infty
$$

uniformly in $s$, then we have a non-autonomous attractor (see Chepyzhov and Vishik 1993), with $\mathcal{A}(t) \subset B$ for all $t \in \mathbb{R}$. (Note that the condition in (3.4) requires attraction to $B$ as $t \rightarrow \infty$, although we only obtain the pullback convergence (3.3) for the non-autonomous attractor itself. This incongruity is rectified in Crauel et al (1995) and Schmalfuß (1999), where the absorbing property is only required to hold in a pullback sense.)

Provided that we take $f(t) \in C_{b}^{0}(\mathbb{R} ; H)$ (continuous and bounded into $H$ ), condition (3.4) holds for the non-autonomous Navier-Stokes equations, and so they have a non-autonomous attractor. Furthermore, it is not hard to show that if $u \in \mathcal{A}(s)$ then

$$
\left|S\left(t_{1}, s\right) u-S\left(t_{2}, s\right) u\right| \leqslant L\left|t_{1}-t_{2}\right| .
$$

The non-autonomous attractor is said to be finite dimensional if each of the kernel sections $\mathcal{A}(t)$ has finite dimension. Chepyzhov and Vishik (1993) show that the kernel sections of 
the non-autonomous Navier-Stokes equation all enjoy the same bound on their Hausdorff dimension.

They note that their results can easily be adapted to treat the case of fractal dimension, but do not give the details. Since it is fundamental to our approach that this stronger measure of dimension is also finite, it seems worthwhile to present the necessary adjustments here. However, in what follows we will only need the end result, and so we relegate our brief treatment to an appendix. With these small changes, the calculations in Chepyzhov and Vishik (1993) guarantee that the kernel sections satisfy, for some $d>0$, the finite-dimensional bound

$$
d_{f}(\mathcal{A}(t))<d \quad \text { for all } t \in \mathbb{R} .
$$

\section{An abstract parametrization for all $t$}

We now prove a similar result to theorem 2.1 for the non-autonomous attractor. Note that (3.1) and (3.5) show that condition (4.1) holds for our example with $\theta=1$.

Theorem 4.1. Let $\mathcal{A}(t)$ be an invariant set as in (3.2) with finite fractal dimension uniform overt,

$$
d_{f}(\mathcal{A}(t))<d .
$$

Assume furthermore that $S(t, s)$ is Hölder continuous with exponent $\theta$, so that

$$
\left|S\left(t_{1}, s\right) u-S\left(t_{2}, s\right) v\right| \leqslant C\left(|u-v|^{\theta}+\left|t_{1}-t_{2}\right|^{\theta}\right) \quad \text { for all } \quad u, v \in H, D t_{1}, t_{2} \geqslant s .
$$

Then, provided that $k>2(1+d) / \theta$, a prevalent set of linear maps from $H$ into $\mathbb{R}^{k}$ are one-to-one between

$$
\bigcup_{t \in \mathbb{R}} \mathcal{A}(t)
$$

and its image, and provide a Hölder continuous parametrization of

$$
\bigcup_{t \in I} \mathcal{A}(t)
$$

for any compact subset I of $\mathbb{R}$.

The content of the theorem is that we can find one map (and a prevalent set will work) which is one-to-one not only on all the 'kernel sections' $\mathcal{A}(t)$, considered individually, but even on the whole collection in (4.2). This happens even though the closure of this union may not be finite dimensional: Chepyzhov and Vishik (1993, section 6 part 1) give an example of a forcing term for the 2D Navier-Stokes equations with which

$$
d_{f}\left(\overline{\bigcup_{t \in \mathbb{R}} \mathcal{A}(t)}\right)=\infty
$$

even though each kernel section has finite fractal dimension.

Proof. For each $n \in \mathbb{N}$ consider the set

$$
\mathbb{A}_{n}=\bigcup_{-n \leqslant t \leqslant n} \mathcal{A}(t)
$$

This is the image of the set $[0,2 n] \times \mathcal{A}(-n)$ under the map

$$
(t, u) \mapsto S(t,-n) u
$$


which is Hölder continuous with exponent $\theta$. Using elementary properties of the fractal dimension (see Falconer (1990) or Eden et al (1994), for example) this implies that

$$
d_{f}\left(\mathbb{A}_{n}\right)<(d+1) / \theta \text {. }
$$

We can now apply theorem 2.1 to deduce that a prevalent set of linear maps $\Lambda_{n}$ from $H$ into $\mathbb{R}^{k}$ are one-to-one between $\mathbb{A}_{n}$ and its image (with Hölder inverse).

Since $\left\{\Lambda_{n}\right\}$ is a countable collection of prevalent sets, it follows (see the discussion before theorem 2.1) that

$$
\Lambda_{\infty}=\bigcap_{n \in \mathbb{N}} \Lambda_{n}
$$

is also prevalent. It is clear that if $L \in \Lambda_{\infty}$ then $L$ is one-to-one between any $\mathbb{A}_{n}$ and its image, and since $\mathbb{A}_{n}$ is compact this implies that $L^{-1}$ provides a continuous parametrization of $\mathbb{A}_{n}$, and so of any set of the form (4.3).

To show that $L$ is one-to-one between the infinite union in (4.2) and its image, suppose the opposite. Then there must be two elements in (4.2) such that $L u=L v$. However, this a contradiction, since we must have $u, v \in \mathbb{A}_{n}$ for some $n$.

\section{Nodal parametrization in the non-autonomous case}

A similar argument enables us to prove a non-autonomous version of theorem 2.2. Provided that we also assume that $f(t)$ is uniformly bounded (with respect to $t$ ) in $D\left(\mathrm{e}^{\sigma A^{1 / 2}}\right)$ for some $\sigma>0$, then the paper of Foias and Temam (1989) already contains the necessary bound for solutions which shows that the kernel sections are all bounded in $G_{\tau}(Q)$,

$$
\left|\mathrm{e}^{\tau A^{1 / 2}} u\right| \leqslant K \quad \text { for all } \quad u \in \mathcal{A}(t)
$$

with $K$ uniform over all $t \in \mathbb{R}$. We can therefore apply the following general result to the non-autonomous 2D Navier-Stokes equations.

Theorem 5.1. Let the conditions of theorem 4.1 hold, and in addition assume that $\mathcal{A}(t)$ is uniformly bounded (with respect to $t$ ) in $G_{\tau}(Q)$. Then, provided that $k \geqslant[16(d+1) / \theta]+1$, almost every choice of $k$ nodes $\left(x_{1}, \ldots, x_{k}\right)$ in $Q^{k}$ makes the map $E_{x}[\cdot]$ from (2.4) one-to-one between

$$
\bigcup_{t \in \mathbb{R}} \mathcal{A}(t)
$$

and its image, and provides a parametrization of

$$
\bigcup_{t \in I} \mathcal{A}(t)
$$

for any compact subset I of $\mathbb{R}$.

Proof. As in the proof of theorem 4.1 we consider the sets

$$
\mathbb{A}_{n}=\bigcup_{-n \leqslant t \leqslant n} \mathcal{A}(t)
$$

Each of these satisfies the hypotheses of theorem 2.2, and so almost every choice of $k$ nodes is one-to-one between $\mathbb{A}_{n}$ and its image. As before, since the intersection of a countable collection of sets with full measure still has full measure, almost every choice of $k$ nodes is one-to-one on (5.1) and provides a parametrization of every set of the form (5.2). 


\section{Random dynamical systems}

These results are also applicable to the case of random attractors (see the references at the start of section 3). We will not choose to treat this case in detail here, since a proper summary of the ideas would be much longer than the result itself. Very briefly, for a random dynamical system $\left(\varphi(t, \omega), \theta_{t}\right)$ (see Arnold (1998) for details), a random attractor is a random compact set $\mathcal{A}(\omega)$ such that

$$
\varphi(t, \omega) \mathcal{A}(\omega)=\mathcal{A}\left(\theta_{t} \omega\right)
$$

and, for any bounded set $D$,

$$
\operatorname{dist}\left(\varphi\left(t, \theta_{-t} \omega\right) D, \mathcal{A}(\omega)\right) \rightarrow 0 \quad \text { as } \quad t \rightarrow \infty .
$$

(Compare these with (3.2) and (3.3).) Since a result of Debussche (1998) provides a bound on the fractal dimension of such attractors, application of the results of section 4 gives, for each fixed $\omega$, a prevalent set of linear maps which are one-to-one between

$$
\bigcup_{t \in \mathbb{R}, \omega \in W} \mathcal{A}\left(\theta_{t} \omega\right)
$$

for any countable collection $W \subset \Omega$ (the sample space) and its image (and a parametrization of the union over any compact subset of $\mathbb{R}$ and finite collection of $\omega$ ). We hope to investigate the reduction of such stochastic flows to finite-dimensional stochastic differential equations using these ideas.

The 'instantaneous determining nodes' result of theorem 5.1 should also generalize for examples in which it is possible to prove that the attractor consists of functions in some Gevrey class. Chueshov (2000) has shown this for a stochastic reaction-diffusion equation and for the 2D stochastic Navier-Stokes equations (private communication). It follows again that values of $u$ at a sufficiently large collection of nodes will distinguish functions in

$$
\bigcup_{t \in \mathbb{R}, \omega \in W} \mathcal{A}\left(\theta_{t} \omega\right)
$$

where $W$ is any countable subset of the sample space $\Omega$.

\section{Conclusion}

We have removed the restriction to the autonomous case for two results which concern the modelling of fluid flows with a finite number of degrees of freedom, showing that they apply to the more physically relevant case of a time-dependent forcing. (Of course, the results also apply to other systems which satisfy the conditions of our theorems: Friz and Robinson (2001) contains many examples.) Although we have treated the case of periodic boundary conditions for simplicity, similar results are true for Dirichlet boundary conditions (see Friz et al (2001) for the corresponding results in the autonomous case).

\section{Acknowledgments}

JCR is a Royal Society University Research Fellow, and would like to thank the Society for their support. Also he would like to thank the Departamento EDAN in Seville for their hospitality, and Iberdrola for their generous financial assistance during his stay there. JAL has been partially supported by Proyecto DGICYT PB98-1134. 


\section{Appendix}

Since the adjustments are minimal, we do not state all the assumptions, which would take longer than our brief 'proof', and refer to Chepyzhov and Vishik (1993) for the details. We want to show that their theorem 3.1 holds, replacing their (26) by

$$
d_{f}\left(X_{i}\right) \leqslant 2 d \quad \text { for all } i \in \mathbb{Z} .
$$

To do this we follow the argument of theorem 3.2 in chapter $\mathrm{V}$ of Temam (1988), making the same adjustments as used by Chepyzhov and Vishik for the Hausdorff dimension, until we reach part (iii) of Temam's proof, having deduced that

$$
\phi_{i+1}(\alpha \epsilon) \leqslant \frac{1}{2} \phi_{i}(\epsilon) \quad \text { for all } \quad 0<\epsilon \leqslant \epsilon_{0}
$$

where now

$$
\phi_{j}(\epsilon)=\epsilon^{d} N\left(X_{j}, \epsilon\right)
$$

We also write

$$
\bar{\phi}(\epsilon)=\epsilon^{d} N(B, \epsilon)
$$

where $B$ is the compact absorbing set from (3.4), so that we have $X_{i} \subset B$ for all $i$.

We choose $j=j(\epsilon)$ as in Temam, with $\alpha^{j+1} \leqslant \epsilon<\alpha^{j}$, and then we have

$$
\phi_{i}(\epsilon)=\phi_{i}\left(\alpha^{j} \alpha^{-j} \epsilon\right) \leqslant 2^{-j} \phi_{i-j}\left(\alpha^{-j} \epsilon\right) .
$$

Setting

$$
\begin{aligned}
M & =\sup _{i-j \leqslant k \leqslant i} \sup _{\alpha \epsilon_{0} \leqslant \epsilon^{\prime} \leqslant \epsilon_{0}} \phi_{k}\left(\epsilon^{\prime}\right) \leqslant \sup _{\alpha \epsilon_{0} \leqslant \epsilon^{\prime} \leqslant \epsilon_{0}} \bar{\phi}\left(\epsilon^{\prime}\right) \\
& =\sup _{\alpha \epsilon_{0} \leqslant \epsilon^{\prime} \leqslant \epsilon_{0}}\left(\epsilon^{\prime}\right)^{d} N\left(X, \epsilon^{\prime}\right) \leqslant \epsilon_{0}^{d} N\left(X, \alpha \epsilon_{0}\right)<\infty
\end{aligned}
$$

we obtain

$$
\phi_{i}(\epsilon) \leqslant 2^{-j} M
$$

and so $\phi_{i}(\epsilon) \rightarrow 0$ as $\epsilon \rightarrow 0$. It now follows as in Temam (1988) that $d_{f}\left(X_{i}\right) \leqslant 2 d$ for every $i$.

\section{References}

Arnold L 1998 Random Dynamical Systems (Berlin: Springer)

Chepyzhov V and Vishik M 1993 A Hausdorff dimension estimate for kernel sections of non-autonomous evolution equations Indiana Univ. Math. J. 42 1057-76

1994 Attractors of non-autonomous dynamical systems and their dimension J. Math. Pure. Appl. 73 279-333

Chueshov I D 2000 Gevrey regularity of random attractors for stochastic reaction-diffusion equations Random Operators Stoch. Eq. 8 143-62

Cockburn B, Jones D A and Titi E S 1997 Estimating the number of asymptotic degrees of freedom for nonlinear dissipative systems Math. Comput. 66 1073-87

Constantin P and Foias C 1985 Global Lyapunov exponents, Kaplan-Yorke formulas and the dimension of the attractor for 2D Navier-Stokes equation Commun. Pure Appl. Math. 38 1-27

1988 Navier-Stokes Equations (Chicago, IL: University of Chicago Press)

Crauel H, Debussche A and Flandoli F 1995 Random attractors J. Dynam. Diff. Eq. $9307-41$

Crauel H and Flandoli F 1994 Attractors for random dynamical systems Prob. Theor. Relat. Fields 100 365-93

Debussche A 1998 Hausdorff dimension of a random invariant set J. Math. Pure. Appl. 77 967-88

Doering C R and Gibbon J D 1995 Applied Analysis of the Navier-Stokes Equations (Cambridge: Cambridge University Press) 
Eden A, Foias C, Nicolaenko B and Temam R 1994 Exponential Attractors for Dissipative Evolution Equations (New York: Wiley)

Falconer K 1990 Fractal Geometry (Chichester: Wiley)

Foias C and Prodi G 1967 Sur le comportement global des solutions non stationnaires des équations de Navier-Stokes en dimension 2 Rend. Sem. Mat. Univ. Padova 39 1-34

Foias C, Sell G R and Temam R 1988 Inertial manifolds for nonlinear evolution equations J. Diff. Eq. 73 309-53

Foias C and Temam R 1984 Determination of the solutions of the Navier-Stokes equations by a set of nodal values Math. Comput. 43 117-33

1989 Gevrey class regularity for the solutions of the Navier-Stokes equations J. Funct. Anal. 87 359-69

Foias C and Olson E J 1996 Finite fractal dimensions and Hölder-Lipschitz parametrization Indiana Univ. Math. J. 45 603-16

Friz P K, Kukavica I and Robinson J C 2001 Nodal parametrisation of analytic attractors Discrete Continuous Dynam. Syst. 7 643-57

Friz P K and Robinson J C 2001 Parametrising the attractor of the two-dimensional Navier-Stokes equations with a finite number of nodal values Physica D 148 201-20

Hale J K 1988 Asymptotic Behaviour of Dissipative Systems (Mathematics Surveys and Monographs vol 25) (Providence, RI: American Mathematical Society)

Hunt B R and Kaloshin V Y 1999 Regularity of embeddings of infinite-dimensional fractal sets into finite-dimensional spaces Nonlinearity 12 1263-75

Hunt B R, Sauer T and Yorke J A 1992 Prevalence: a translation-invariant almost every for infinite dimensional spaces Bull. Am. Math. Soc. 27 217-38

1993 Prevalence: an addendum Bull. Am. Math. Soc. 28 306-7

Kloeden P and Schmalfuß B 1997 Nonautonomous systems, cocycle attractors and variable time-step discretization Numer. Algorithms 14 141-52

Ladyzhenskaya O A 1991 Attractors for Semigroups and Evolution Equations (Cambridge: Cambridge University Press)

Mañé 1981 On the Dimension of the Compact Invariant Sets of Certain Nonlinear Maps (Springer Lecture Notes in Mathematics vol 898) (Berlin: Springer) pp 230-42

Robinson J C 1999 Global attractors: topology and finite-dimensional dynamics J. Dynam. Diff. Eq. 11 557-81

-2001a A rigorous treatment of experimental observations for the two-dimensional Navier-Stokes equations Proc. R. Soc. A 457 1007-20

-2001b Infinite-Dimensional Dynamical Systems (Cambridge: Cambridge University Press)

Schmalfuß B 1992 Backward cocycles and attractors of stochastic differential equations Int. Seminar on Applied Mathematics - Nonlinear Dynamics: Attractor Approximation and Global Behaviour ed V Reitman, T Redrich and N Kosch pp 185-92

2000 Attractors for the non-autonomous dynamical systems Proc. Equadiff 99 (Berlin) ed B Fielder, K Gröger and J Sprekels (Singapore: World Scientific) pp 684-9

Temam R 1988 Infinite-Dimensional Dynamical Systems in Mechanics and Physics (Berlin: Springer, Providence, RI: American Mathematical Society) vol 68 (1996 2nd edn) 